\title{
A study of an orbital cycle of GX 301-2 observed by BeppoSAX
}

\author{
A. La Barbera ${ }^{1}$, A. Segreto ${ }^{1}$, A. Santangelo ${ }^{1,2}$, I. Kreykenbohm ${ }^{2,3}$, and M. Orlandini ${ }^{4}$ \\ ${ }^{1}$ Istituto di Astrofisica Spaziale e Fisica Cosmica, INAF, Sezione di Palermo, via Ugo La Malfa 153, 90146 Palermo, Italy \\ e-mail: nino. labarbera@pa.iasf.cnr.it \\ 2 Institut für Astronomie und Astrophysik - Astronomie, Sand 1, 72076 Tübingen, Germany \\ 3 INTEGRAL Science Data Centre, 6 Ch. d'Écogia, 1290 Versoix, Switzerland \\ ${ }^{4}$ Istituto di Astrofisica Spaziale e Fisica Cosmica, INAF, Sezione di Bologna, via Piero Gobetti 101, 40129 Bologna, Italy
}

Received 22 June 2004 / Accepted 15 March 2005

\begin{abstract}
In this paper we report on the 4-80 keV wide-band X-ray spectrum of the High Mass X-ray Binary GX 301-2 observed by the BeppoSAX satellite during an extended monitoring campaign in 1998. The source was observed at 4 different orbital phases corresponding to diverse luminosity states. The $4-80 \mathrm{keV}$ continuum is described well by the multi component partial-covering absorber model corrected by a high energy cutoff. In addition to the $\mathrm{K}_{\alpha}$ (at $\sim 6.4 \mathrm{keV}$ ) iron emission fluorescence line, the $\mathrm{K}_{\beta}$ (at $\sim 7 \mathrm{keV}$ ) was observed together with an absorption-like feature at hard energies that we interpret as a cyclotron resonance scattering feature (CRSF). Both the continuum and the CRSF show clear dependence on the orbital phase. This is likely to be caused by the variation in the luminosity with the orbit, even if the resulting relation between cyclotron energy and luminosity is difficult to interpret within the present one-dimensional models of accretion columns. In fact, when the neutron star is close to periastron and the system is in a low luminosity state $\left(L_{\mathrm{x}} \sim 0.8 \times 10^{37} \mathrm{erg} / \mathrm{s}\right.$ for an assumed distance of $\left.1.8 \mathrm{kpc}\right)$, the CRSF centroid energy is observed at $\sim 45 \mathrm{keV}$, while at orbital phase $\sim 0.9$ (at a maximum luminosity of $\sim 2 \times 10^{37} \mathrm{erg} / \mathrm{s}$ ) it is $53 \mathrm{keV}$ in contrast with predictions. The cyclotron energy is also found to vary with the pulse phase, asymmetrically with respect to the pulse profile. Consequences for the geometry of the magnetic field are finally discussed.
\end{abstract}

Key words. pulsars: individual: GX 301-2 - stars: neutron - stars: magnetic fields - X-rays: binaries

\section{Introduction}

GX 301-2 is a High Mass X-ray Binary system (HMXB) consisting of a neutron star orbiting a B2 Iae star (WRA 977), which has already evolved off the main sequence and whose age has been estimated to about $10^{6} \mathrm{yr}$. The distance to the system is still under discussion; while spectroscopic observations seem to suggest a distance of $1.8 \pm 0.4 \mathrm{kpc}$ (Parkes et al. 1980), Kaper et al. (1995) have estimated a distance of $5.3 \mathrm{kpc}$. Taking into account both estimates, the mass loss rate should range between $3 \times 10^{-6}$ and $1 \times 10^{-5} M_{\odot} / \mathrm{yr}$.

The orbital period of the system is $\sim 41.5$ days (White et al. 1978; Sato et al. 1986; Koh et al. 1997). Coherent X-rays pulsations were discovered by the SAS 3 satellite with a period of $\sim 700 \mathrm{~s}$ (White et al. 1976). The X-ray mass function of the system has been estimated to be $\sim 31.9 M_{\odot}$ (Sato et al. 1986; Koh et al. 1997). Assuming that the neutron star has a mass of $1.4 M_{\odot}$, the mass of the companion is $\sim 30.3 M_{\odot}$. Parkes et al. (1980) calculate the photospheric radius of the stellar companion as $43 R_{\odot}$. This value, associated with the absence of eclipse, constrains the inclination of the orbital plane with respect to the line of sight to be less than $78^{\circ}$.
The orbit of GX 301-2 is characterized by high eccentricity $(e \sim 0.46)$. This causes a large change in the mass accretion rate onto the neutron star over the orbit that results in a large X-ray luminosity variations in the range $(2-200) \times 10^{35} \mathrm{erg} \mathrm{s}^{-1}$ producing a periodic outburst. This periodic outburst, first discovered by Watson et al. (1982), appears at an orbital phase $\sim 1.4$ days before the periastron passage of the neutron star (Sato et al. 1986). Batse observations (Pravdo et al. 1995; Koh et al. 1997) suggest a secondary outburst near the apastron passage. Although several models have been proposed to explain these outbursts (see e.g. Haberl 1991; Pravdo \& Ghosh 2001; Leahy 2002), so far there is no general agreement on an explanation for the X-rays intensity modulation during the orbital revolution.

It is thought that GX $301-2$ is a wind fed system. Measurements obtained from the hydrogen Balmer lines at a distance of $R / R_{\mathrm{c}} \sim 3$ (Parkes et al. 1980), where $R_{\mathrm{c}}$ is the radius of the secondary, suggest a terminal wind velocity $v_{\infty}$ of $\sim 400 \mathrm{~km} \mathrm{~s}^{-1}$. In fact the high mass-loss rate associated with the relatively slow wind velocity causes stagnation of a large amount of matter in the binary system. Although the real 
geometry of the accretion is more complex than the simple spherical accretion (e.g. detailed numerical simulations show the formation of an accretion wake trailing the neutron star, Blondin et al. 1990; Blondin et al. 1991), the mass accretion rate agrees with the one predicted by the classical estimate for spherical accretion within a factor 2 .

The X-ray spectrum of GX 301-2 has usually been described by a power law model with a high energy cutoff (White et al. 1983; Makishima \& Mihara 1992; Mihara 1995; Orlandini et al. 2000). The resulting photon index ranges between 1.1 and 1.5, while the cutoff energy ranges between 20 and $26 \mathrm{keV}$ and the folding energy between 6 and $12 \mathrm{keV}$. Based on Ginga data, Mihara et al. (1998) modeled the spectral continuum with the Negative and Positive power laws EXponential model (NPEX) (Mihara 1995), i.e. $\left(E^{\alpha}+E^{\beta}\right) \exp (-E / k T)$, consisting of an intrinsic power law with negative photon index $\alpha$ plus its Comptonized component with $\beta \simeq 2$. The continuum spectrum of GX 301-2 has also been described by a power law undergoing two different photoelectric absorptions described in terms of two hydrogen column densities (partial-covering absorber model); see e.g. Endo et al. (2002) and references therein. Saraswat et al. (1996) interpreted the less absorbed continuum component as produced by matter surrounding the pulsar, while they attributed the highly absorbed component to the emission produced at the neutron star magnetic polar caps. But the detection of significant pulsations also from the less absorbed component in ASCA observations (Endo et al. 2002) seems to exclude this interpretation, indicating that the whole continuum is emitted directly from the neutron star. Recently Kreykenbohm et al. (2004) also used the partial-covering absorber model to fit the spectral continuum of GX 301-2 observed by RXTE.

The low energy X-ray spectrum $(E<4 \mathrm{keV})$ of GX 301-2 is dominated by a strong and variable photoelectron absorption. The hydrogen column density ranges from $\sim 2 \times 10^{23}$ up to $\sim 2 \times 10^{24} \mathrm{~cm}^{-2}$ (see e.g. Leahy et al. 1988), and a strong iron $\mathrm{K}_{\alpha}$ line emission is also evident (White \& Swank 1984). Temna observations of GX 301-2 showed both an iron emission at $\sim 6.4 \mathrm{keV}$ and an iron edge at $\sim 7.4$ (Leahy et al. 1988). The equivalent width of the line varies from 200 to $1800 \mathrm{eV}$. The intensity of the iron $\mathrm{K}_{\alpha}$ line and the $N_{\mathrm{H}}$ are correlated, suggesting that the iron line is emitted via fluorescence from matter of solar abundance surrounding the neutron star. ASCA observations (Endo et al. 2002) seem to suggest that the iron $\mathrm{K}_{\alpha}$ line is emitted via fluorescence from material well inside the accretion radius at a distance within $(1-10) r_{\mathrm{A}}<10^{10} \mathrm{~cm}$ where $r_{\mathrm{A}}$ is the Alfvén radius. Endo et al. (2002) claim the presence of a broad wing component in the iron $\mathrm{K}_{\alpha}$ line profile during the periastron passage which is modulated according to the spin phase of the pulsar. They interpret this component as being composed of the blue and redshifted $\sim 6.4 \mathrm{keV}$ iron lines emitted from matter approaching and receding in the accretion column. Even if the iron line is produced in a region very close to the neutron star, there is some evidence that the ionization state of the iron is only up to Fe IV. The $\mathrm{K}_{\beta}$ emission line was first discovered in the ASCA observations (Endo et al. 2002), confirming that the ionization state of the iron in the fluorescing matter and in the line of sight are consistent with each other and close to being neutral.

The presence of a cyclotron absorption line at $\sim 37 \mathrm{keV}$ was first been claimed by Makishima \& Mihara (1992) based on Ginga observations, and later confirmed by the BeppoSAX observations (Orlandini et al. 2000). It is thought that this kind of high energy absorption feature, observed in a large number of HMXBs, is produced by the resonant scattering of electrons in the Landau levels. Therefore cyclotron resonant scattering features (CRSFs) provide a powerful tool for directly measuring the magnetic field strengths of accreting neutron stars in X-ray binaries. Magnetic fields in X-ray pulsars are typically on the order of $10^{12-13}$ Gauss. Since the electron cyclotron energy $E_{\text {cycl }} \simeq 11.6 B_{12} \mathrm{keV}$, where $B_{12}$ is the magnetic field strength in unit of $10^{12}$ Gauss, CRSFs are expected to be observed at energies higher than $10 \mathrm{KeV}$.

The X-ray emission processes of accreting HMXBs are thought to be strongly influenced by their magnetic fields. The knowledge of the magnetic field strength and its structure is, therefore, crucial to understanding the physics and geometry of the X-ray emission from HMXB (Nagel 1981; Meszaros \& Nagel 1985a,b; Araya \& Harding 1999, 2000).

The main aim of this paper is to study the high energy (4-80 keV) spectrum of GX 301-2 as a function of the orbital motion and of the pulse phase, based on several BeppoSAX observations. Particular attention has been given to the study of the iron fluorescence line, in order to analyze the state of the matter surrounding the neutron star, and to compare our results to those of ASCA. The CSRF was also investigated in detail, permitting a better description of the geometric structure of the magnetic field around the neutron star and consequently a deeper understanding of the accretion column physics.

\section{BeppoSAX observations and data reduction}

The BeppoSAX satellite (Boella et al. 1997a) was active from April 1996 untill April 2002. The payload was composed of four Narrow Field Instrument (NFIs) and two Wide Field Cameras (Jager et al. 1997). Besides the Low-Energy Concentrator Spectrometer (LECS, 0.1-10 keV, Parmar et al. 1997) and the Medium-Energy Concentrator Spectrometer (MECS, 2-10 keV, Boella et al. 1997b), the NFIs consist of the two collimated high energy detectors, the High Pressure Gas Scintillation Proportional Counter (HPGSPC, 4-60 keV, FWHM energy resolution of $8 \%$ at $10 \mathrm{keV}$ and $5.5 \%$ at $20 \mathrm{keV}$, Manzo et al. 1997), and the Phoswich Detection System (PDS, 15-200 keV, FWHM energy resolution of $24 \%$ at $20 \mathrm{keV}$, and $14 \%$ at $60 \mathrm{keV}$, Frontera et al. 1997). The two collimated instruments (HPGSPC and PDS) were operated in rocking mode with a 96 s stay time in order to monitor the background along the orbit. Details on data reduction and spectral analysis can be found in the "Cookbook for BeppoSAX NFI spectral analysis" (Fiore et al. 1999).

The BeppoSAX satellite observed GX 301-2 with NFIs in several orbital phase intervals from January 7, 1998 to January 30, 1998. In Table 1 we report the list of BeppoSAX observations of GX 301-2 and the relative orbital phases. We divided our observations (NFI OP) into 4 orbital phase 
Table 1. Log of the BeppoSAX observations of GX 301-2.

\begin{tabular}{lllll}
\hline \hline Phase Group & NFI OP & Start time & $\begin{array}{l}\text { Obs. time } \\
(\mathrm{ks})\end{array}$ & Orbit. phase $^{a}$ \\
\hline Postapastron & 3275 & Jan. 07 1998 14:46:40 & 96 & $0.62-0.65$ \\
Preperiastron & 3373 & Jan. 17 1998 22:18:43 & 85 & $0.87-0.90$ \\
Periastron & 3428 & Jan. 23 1998 13:02:57 & 100 & $0.008-0.036$ \\
Periastron & 3429 & Jan. 24 1998 16:49:37 & 27 & $0.036-0.044$ \\
Intermediate & 3503 & Jan. 30 1998 07:27:02 & 15 & $0.17-0.175$ \\
Intermediate & 3514 & Jan. 30 1998 18:29:37 & 60 & $0.18-0.20$ \\
\hline
\end{tabular}

${ }^{a}$ Ephemerides from Koh et al. (1997).

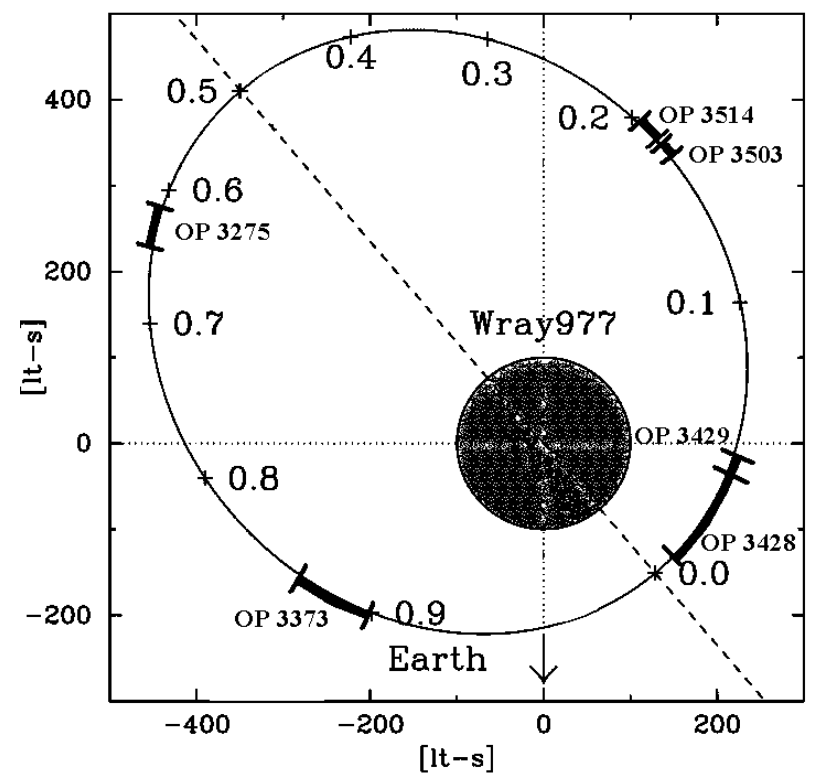

Fig. 1. Orbital phases of the BeppoSAX observations of GX 301-2. The orbital parameters are taken from Koh et al. (1997).

groups, corresponding to 4 different orbital phase intervals: Postapastron, Preperiastron, Periastron, Intermediate (see also Fig. 1).

\section{Time analysis}

Figure 2 shows the average flux observed by the MECS (4-10 keV) and PDS (15-80 keV) in the different orbital phase intervals compared to the folded orbital light curve based on RXTE/ASM data (ephemerides from Koh et al. 1997). The flux levels of GX 301-2 over the orbit in the BeppoSAX observations are in agreement with the mean general trend observed by RXTE/ASM.

We studied the time variability of each selected orbital phase (see Table 1). In Fig. 3 we plot the background subtracted light curves in the $2-10 \mathrm{keV}$ band (MECS) and in the $15-80 \mathrm{keV}$ band (PDS). The low energy and high energy trends are consistent. During the Postapastron observation we observed a flaring episode lasting $\sim 11 \mathrm{~h}$ with the MECS flux varying from less than $1 \mathrm{cts} / \mathrm{s}$ to more than $\sim 7 \mathrm{cts} / \mathrm{s}$. This flare is followed by an episode of quiescence where the flux decreases to $\sim 1 \mathrm{cts} / \mathrm{s}$. During the Preperiastron observation the MECS flux is fairly stable ranging from $\sim 8$ to $\sim 20 \mathrm{cts} / \mathrm{s}$. During the

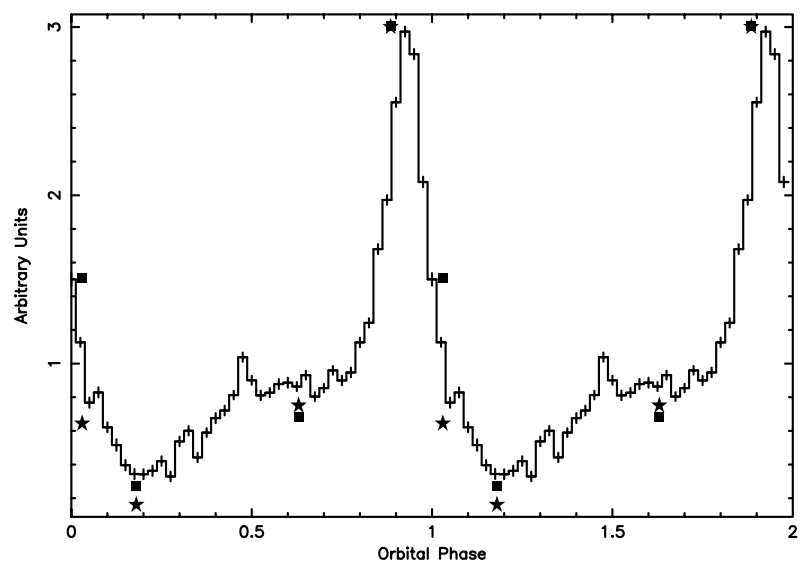

Fig. 2. Mean flux observed by the MECS (4-10 keV) (stars) and by the PDS (15-80 keV) (squares) at the selected orbital phase intervals compared to the folded orbital light curve obtained by the RXTE/ASM data (solid line) from 1996 to 2004. The data have been normalized to permit comparison. Ephemerides are from Koh et al. (1997), phase 0 with the periastron passage. Starting from the left, the selected orbital phase intervals are Periastron, Intermediate, Postapastron, and Preperiastron.

Periastron observation we can distinguish 3 intervals. In the first the mean MECS flux is $\sim 3 \mathrm{cts} / \mathrm{s}$. This interval is followed by a flaring episode where the flux increases up to $\sim 12 \mathrm{cts} / \mathrm{s}$. A quiescent period follows with a flux of $\sim 1 \mathrm{cts} / \mathrm{s}$. During the Intermediate phase the MECS flux is stable around $\sim 0.75 \mathrm{cts} / \mathrm{s}$.

We searched for the spin period of GX 301-2 in all selected orbital phases, obtaining a mean value, corrected for orbital effects, of $679.5 \pm 0.5$ s. In Figs. 4-7, we plot the background subtracted pulse profiles of GX 301-2 during the different orbital phases in some energy ranges: MECS, 2-5 keV and 5-10 keV; HPGSPC, 7-15 keV and 15-30 keV; PDS, 15-30 keV and $30-80 \mathrm{keV}$. Generally the low energy (2-5 keV) pulse profile varies with the orbital phase. The low energy pulse profiles relative to the Postapastron and Preperiastron data $(2-5 \mathrm{keV})$ show a double-peaked structure with two large peaks that also have two minor structures at the top evolving in more feature at higher energies $(5-10 \mathrm{keV})$. Because of the strong absorption it is quite impossible to distinguish the double-peaked structure in the $2-5 \mathrm{keV}$ band of the Periastron data, while it is observable in the 5-10 keV energy band. Because of the low statistics, it is impossible to distinguish the minor structures in 

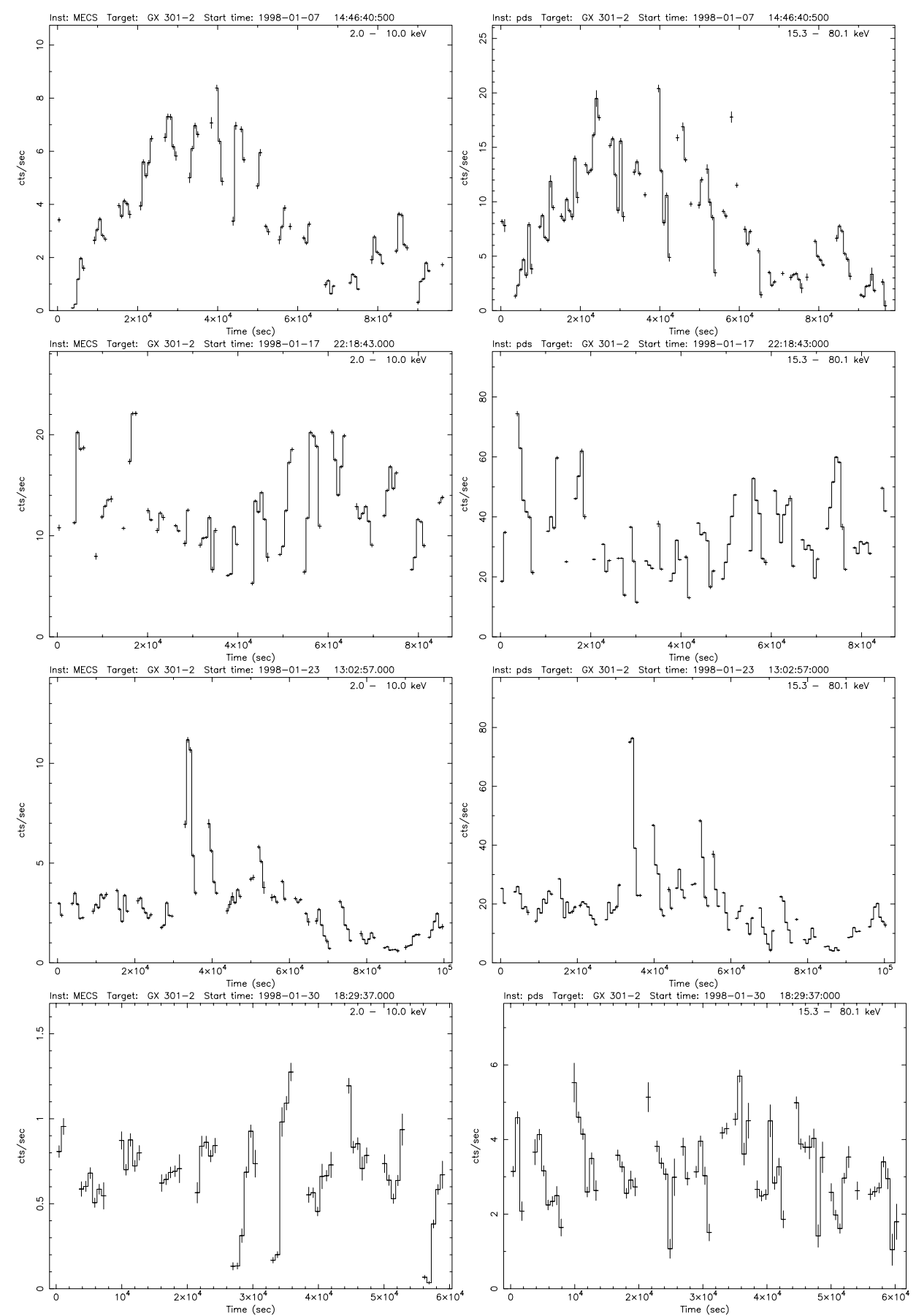

Fig. 3. Background subtracted light curves of GX 301-2. In the left panels the MECS data in the band $2-10 \mathrm{keV}$ are reported. In the right panels we plot the PDS data in the band $15-80 \mathrm{keV}$ for comparison. From the top: Postapastron, Preperiastron, Periastron, and Intermediate.

the double-peaked pulse profile of the Intermediate phase both in the energy intervals $2-5 \mathrm{keV}$ and $5-10 \mathrm{keV}$. This doublepeaked structure is evident at higher energies in the HPGSPC data $(7-15 \mathrm{keV}, 15-30 \mathrm{keV})$ and in the PDS data $(15-30 \mathrm{keV}$, $30-80 \mathrm{keV})$. Generally in the PDS data the two peaks are narrower compared to those of lower energy and do not show the minor features at the top (see e.g. Frontera \& Dal Fiume 1997). A particular pulse profile is observed during the Periastron phase. The PDS data show a strong decrease in the pulsed fraction compared to that observed in the other orbital phases. In addition the shape of the pulsed profile is very different, exhibiting two peaks with large bases and narrow tops.

\section{Averaged spectral analysis}

To understand the evolution of the X-ray spectrum of GX 301-2 over the orbit, we studied the spectra separately corresponding to the different orbital phases. We studied those spectra in the range $4-80 \mathrm{keV}$ obtained by MECS, HPGSPC, and PDS. Below $4 \mathrm{keV}$ the spectrum is completely dominated 

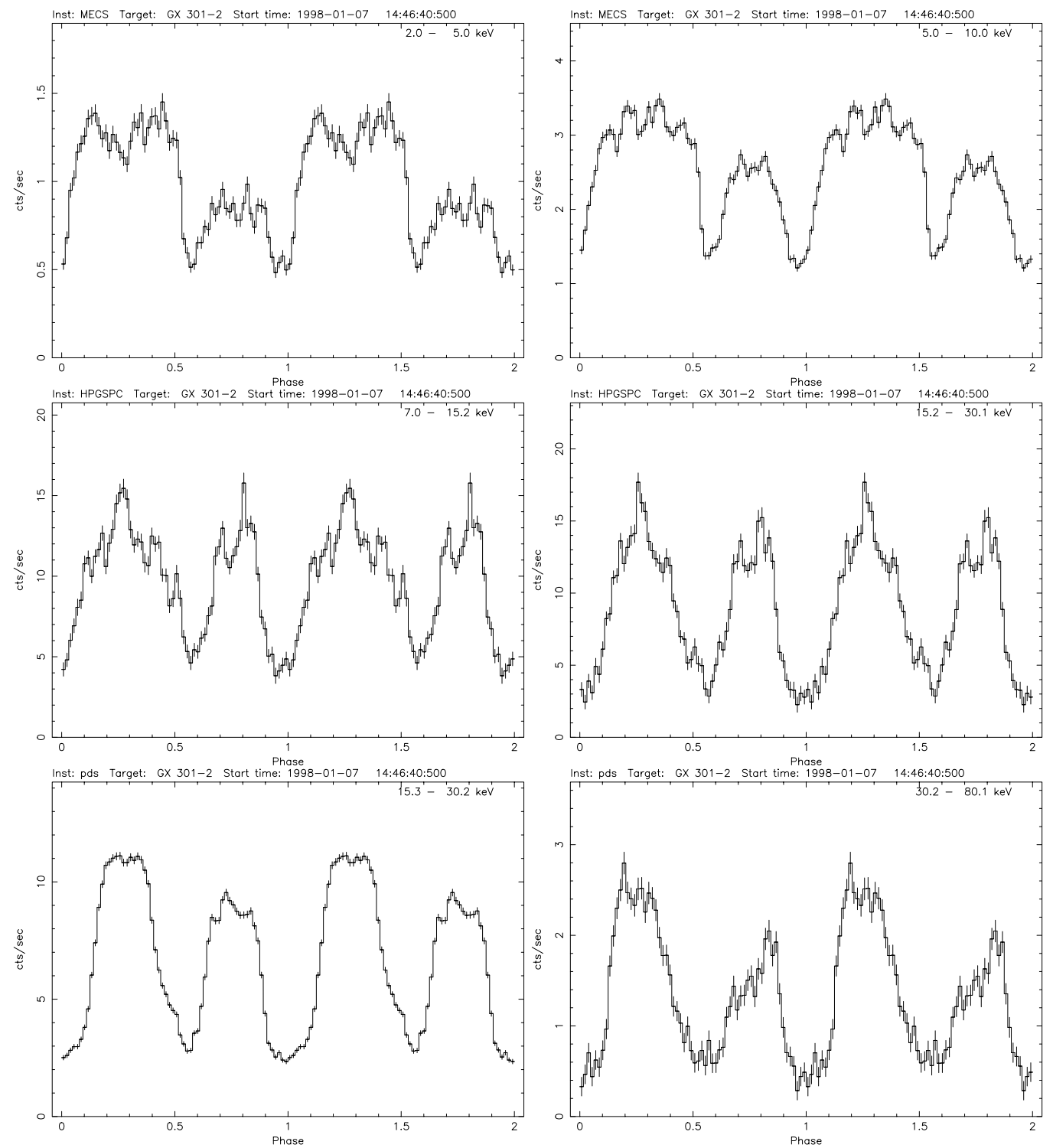

Fig. 4. Background subtracted pulse profile of GX 301-2 during the Postapastron. Top: left panel: MECS 2-5 keV, right panel: MECS 5$10 \mathrm{keV}$; middle: left panel: HPGSPC 7-15 keV, right panel: HPGSPC 15-30 keV; bottom: left panel: PDS 13-30 keV, right panel: 30-80 keV.

by the photoelectric absorption, which is highly time variable, thus making the LECS data useless for determining the spectral continuum shape, whereas above $80 \mathrm{keV}$ the data become statistically meaningless. For our analysis we considered the following energy ranges: 4-10 keV for the MECS, 8-25 keV for the HPGSPC, and finally $15-80 \mathrm{keV}$ for the PDS. In Fig. 8 (left panels) we plot the spectra relative to the selected orbital phase intervals with the residuals from the best fits.

THE CONTINUUM: several models were tested to fit the 4-80 keV spectral continuum including, for instance, a single absorbed power law with high energy cutoff and NPEX. The one that better describes data of Postapastron, Preperiastron, and Periastron phase intervals was the partial-covering absorber model corrected by a high energy cutoff. This model consists of two power law components with a common photon index but they are absorbed by two different hydrogen column densities. To give a general idea in Fig. 9 we plot the unfolded spectrum of GX 301-2 relative to the Preperiastron data. The two components, the less and the more absorbed, which contribute to the spectral continuum, are also represented. The Intermediate data, however, can be also fitted by using a single absorbed power law with high energy cutoff.

To describe the partial-covering absorber model, corrected for the high energy cutoff, we have assumed the following mathematical form:

$f(E)=\operatorname{NHIGH}(E) \times\left(N_{1} w a b s_{1}+N_{2} w a b s_{2}\right) \times E^{-\alpha}$

where $w a b s_{1}$ and $w a b s_{2}$ are the two photoelectric absorption components and $\mathrm{NHIGH}(\mathrm{E})$ is the high energy cutoff correction. To avoid cusps we did not use the XSPEC multiplicative model HIGHECUT but instead used a slightly modified multiplicative model, NHIGH, which is 1 for $E \leq E_{\text {cut }}-W / 2$ but is $\exp \left[-\left[\left(E-E_{\text {cut }}\right) / E_{\text {fold }}\right]\right]$ for $E \geq E_{\text {cut }}+W / 2$. The energy interval of width $W$ between $E_{\text {cut }}-W / 2$ and $E_{\text {cut }}+W / 2$ is connected to the external intervals by a cubic polynomial in order to have 

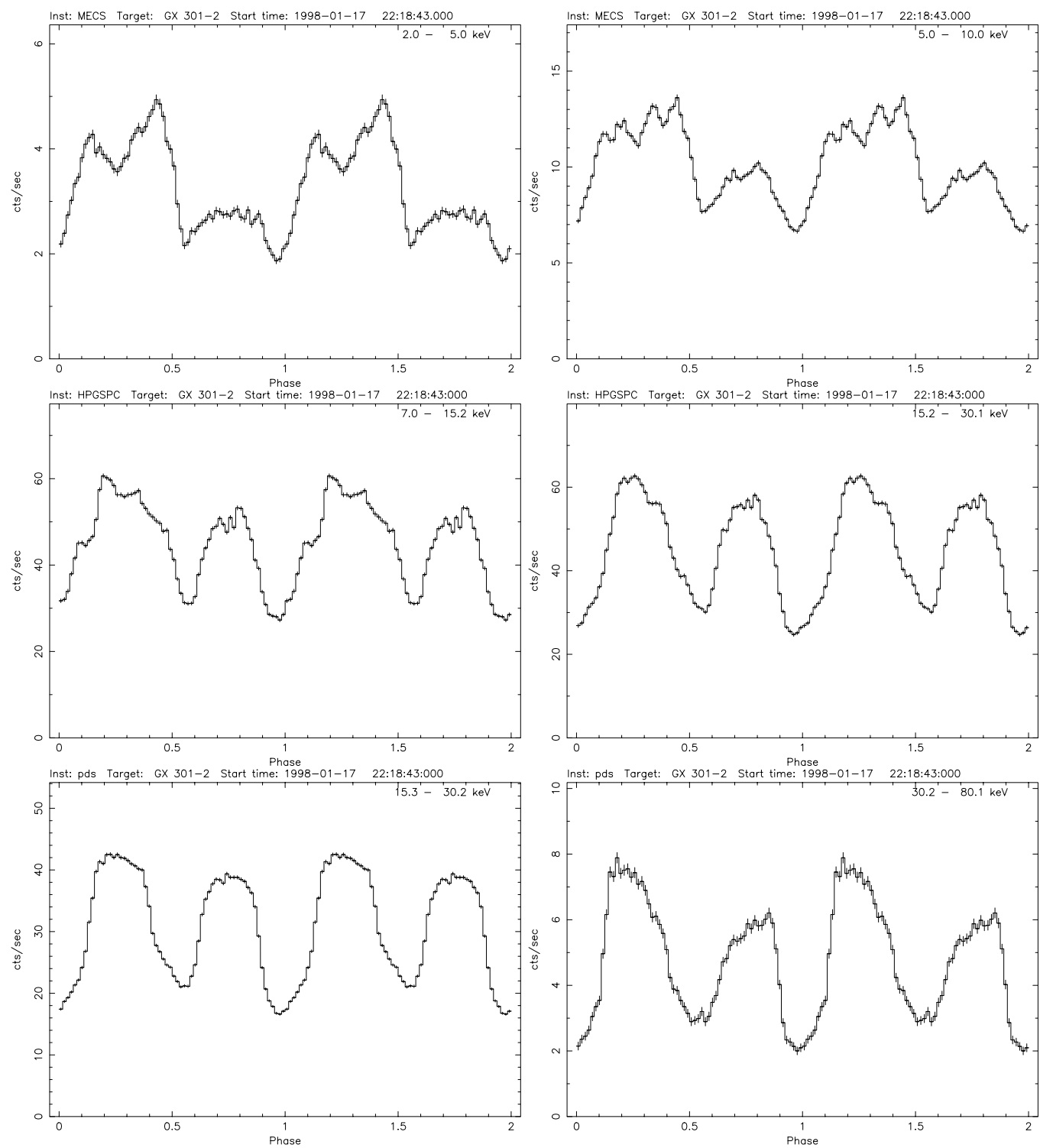

Fig. 5. Background subtracted pulse profile of GX 301-2 during the Preperiastron. Top: left panel: MECS 2-5 keV, right panel: MECS 5$10 \mathrm{keV}$; middle: left panel: HPGSPC 7-15 keV, right panel: HPGSPC 15-30 keV; bottom: left panel: PDS 13-30 keV, right panel: 30-80 keV.

a continuous and derivable mathematical function in the whole energy range (see e.g. Burderi et al. 2000 and La Barbera et al. 2003). The results of the best fits are reported in Table 2.

The spectral photon index is fairly stable over the orbit, ranging from $\sim 0.85$ at the Periastron to $\sim 1.35$ at the Postapastron. Also the cutoff energy $\left(E_{\text {cut }}\right)$ does not show strong variations along the orbit. It ranges from $\sim 19.4 \mathrm{keV}$ at the Periastron to $\sim 22.5 \mathrm{keV}$ in Postapastron and Intermediate data. $E_{\text {cut }}$ results to be smaller near the periastron and larger near the apastron. Similar behavior is observed for the folding energy $\left(E_{\text {fold }}\right)$. In fact this parameter varies from $\sim 8.0 \mathrm{keV}$ (Periastron) to $\sim 10.7 \mathrm{keV}$ (Intermediate). $E_{\text {fold }}$ is smaller near the periastron and increases towards the apastron. The behavior of the two absorbing hydrogen column densities and of normalizations of the power laws is more complex. The less absorbing hydrogen column density is $\sim 2.2 \times 10^{23} \mathrm{~cm}^{-2}$ in the case of the Postapastron and Preperiastron data. This value doubles in the case of the Periastron data. Finally for the Intermediate data, a value of $\sim 5 \times 10^{23} \mathrm{~cm}^{-2}$ is obtained, but it is not possible to directly compare this value with the previous ones because we have used a model with a single power law in this case. The normalization of the power law related to the less absorbing hydrogen column ranges from $4.7 \times 10^{-2}$ photons $/ \mathrm{keV} / \mathrm{cm}^{2} / \mathrm{s}$ at $1 \mathrm{keV}$ (Periastron) to $\sim 0.31$ photons $/ \mathrm{keV} / \mathrm{cm}^{2} / \mathrm{s}$ at $1 \mathrm{keV}$ (Preperiastron). The larger absorbing hydrogen column has values ranging from $11 \times 10^{23} \mathrm{~cm}^{-2}$ (Periastron) to $19 \times 10^{23} \mathrm{~cm}^{-2}$ (Postapastron) but, taking the associated uncertainties into account, these variations are not significant. Normalization of the related power law ranges from $\sim 0.03$ photons $/ \mathrm{keV} / \mathrm{cm}^{2} / \mathrm{s}$ (Postapastron) to $\sim 0.1$ photons $/ \mathrm{keV} / \mathrm{cm}^{2} / \mathrm{s}$ (Preperiastron and Periastron).

THE IRON LINES: a complex emission feature is present between 6 and $7 \mathrm{keV}$ (see Fig. 8, right panels). It is generally 

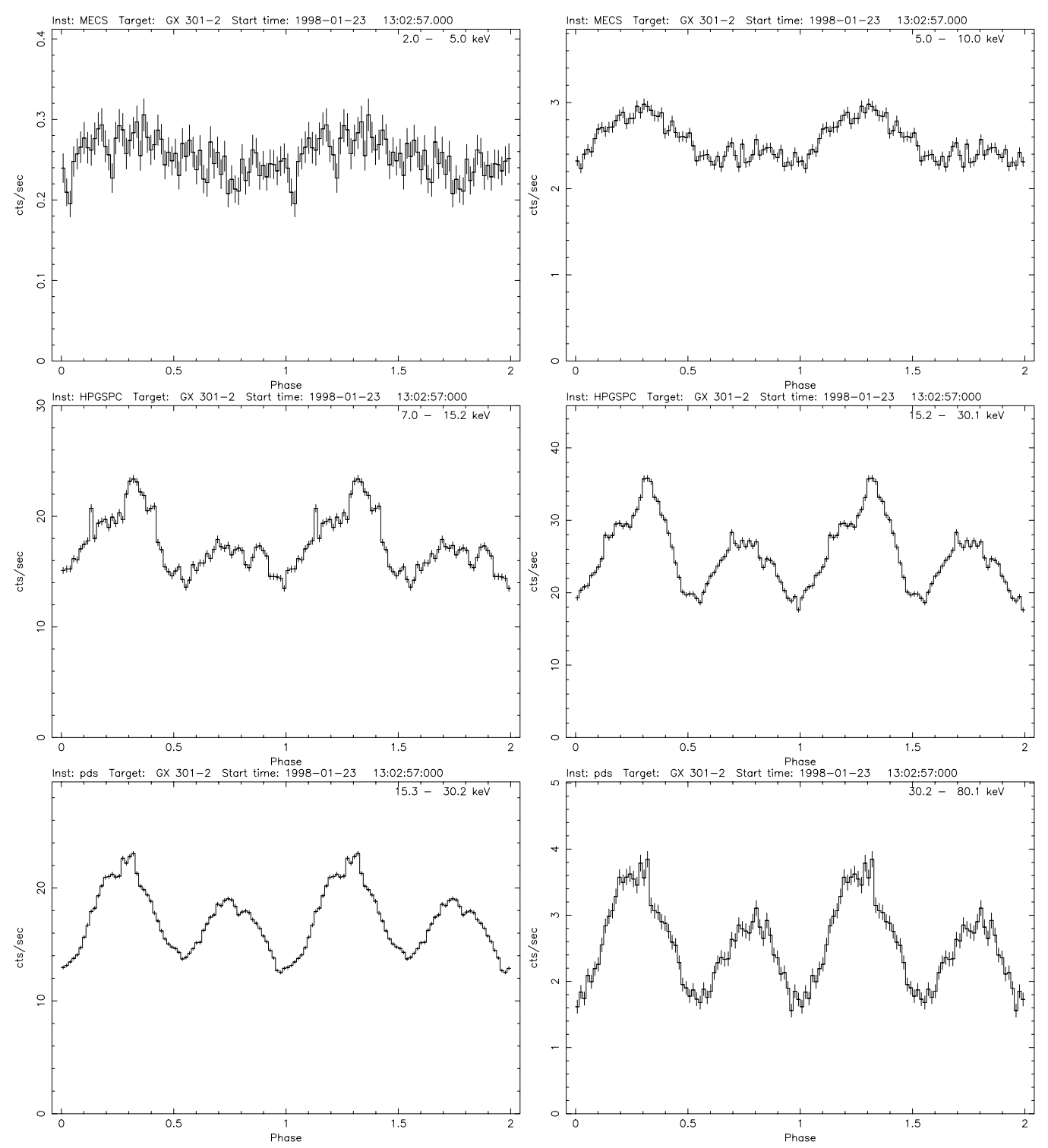

Fig. 6. Background subtracted pulse profile of GX 301-2 during the Periastron. Top: left panel: MECS 2-5 keV, right panel: MECS 5-10 keV; middle: left panel: HPGSPC 7-15 keV, right panel: HPGSPC 15-30 keV; bottom: left panel: PDS 13-30 keV, right panel: 30-80 keV.

thought that this feature is caused by fluorescence from neutral or ionized iron present in the material surrounding the neutron star. For Postapastron, Preperiastron, and Periastron data a single Gaussian is not able to fit the feature. In fact, by fitting with a single Gaussian, the iron line results in a centroid energy of $\sim 6.5 \mathrm{keV}$, whereas its width is $\sim 200 \mathrm{eV}$. But this fitting is unacceptable, obtaining for instance 3.239 (232 d.o.f.) for Preperiastron and 5.803 (230 d.o.f.) for Periastron. By using two Gaussians with the first Gaussian centered at $\sim 6.4 \mathrm{keV}$ and the second centered at $\sim 7.1 \mathrm{keV}$, we obtained an acceptable fit for all orbital phases. The results of the fit of the iron emission feature are reported in Table 2. We interpreted the first Gaussian as the $\mathrm{K}_{\alpha}$ iron line and the second as the $\mathrm{K}_{\beta}$ iron line confirming the ASCA results (Endo et al. 2002). Because of the limited spectral resolution of the MECS we have not been able to precisely estimate the width of the lines. The upper limit for the width of the $\mathrm{K}_{\alpha}$ is $100 \mathrm{eV}$. The Preperiastron data seem to suggest a very tight $\mathrm{K}_{\alpha}$ line less than $35 \mathrm{eV}$. These results are in agreement with those found by ASCA (Endo et al. 2002). A single Gaussian is sufficient to fit the emission line in the Intermediate data, in which case the energy of the centroid is $\sim 6.5 \mathrm{keV}$ and the width is $\sim 250 \mathrm{eV}$. We also used a second component for these data, but the improvement of the fit is statistically not significant. The discrepancy between the results of the Intermediate phase and the other orbital phases is only due to the insufficient statistics which does not permit to correctly resolve the emission line structure. In this sense the line at $6.5 \mathrm{keV}$ is a sort of average of both $\mathrm{K}_{\alpha}$ and $\mathrm{K}_{\beta}$, and it does not correspond to a new ionization state of the iron. No iron edge has been revealed in our data.

THE HIGH ENERGY CYCLOTRON LINE: the spectra of the Preperiastron and Periastron data show an additional absorption feature centered around $\sim 45-50 \mathrm{keV}$, whose presence is independent of the continuum models we used. Such a feature 

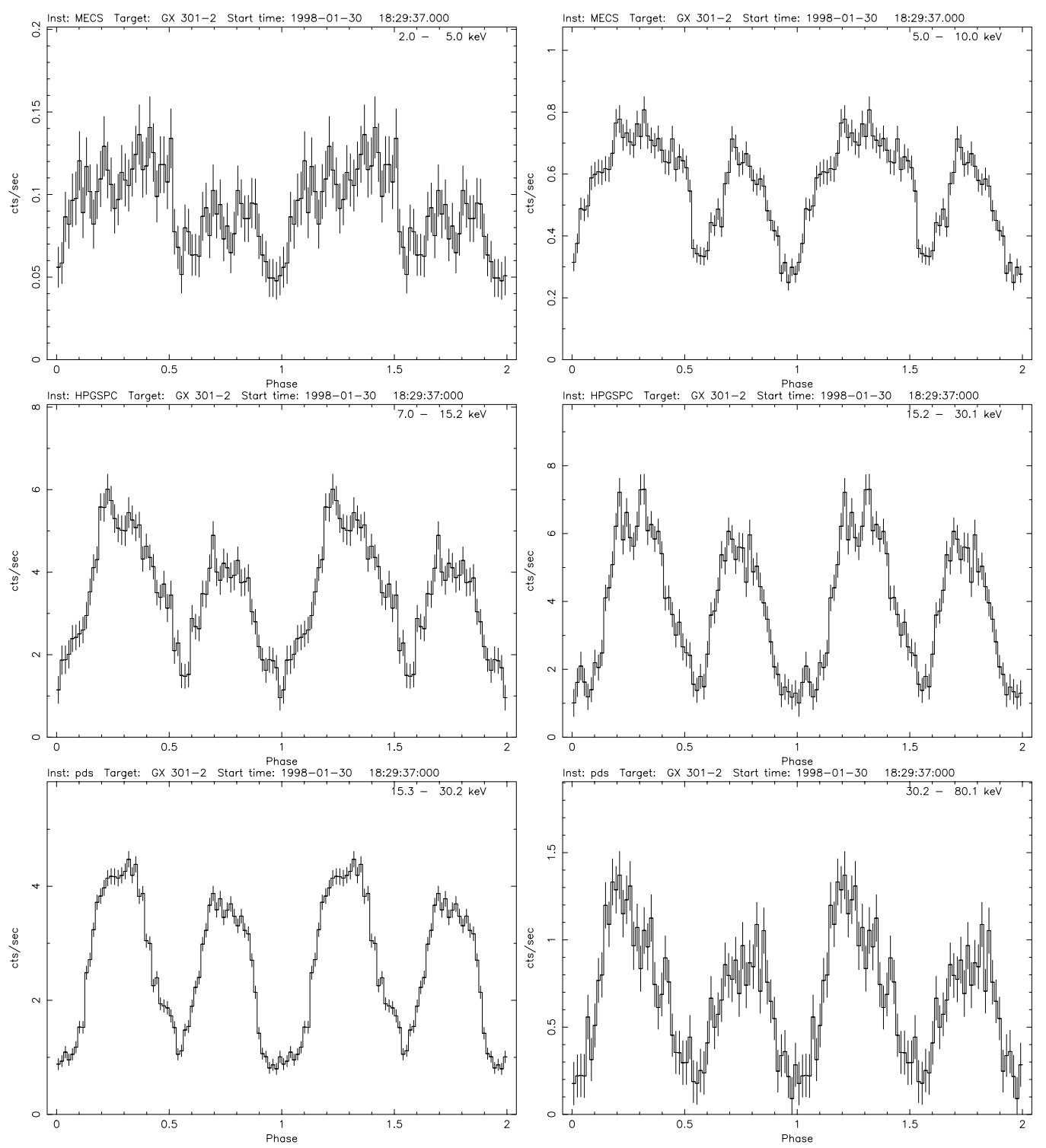

Fig. 7. Background subtracted pulse profile of GX 301-2 during the Intermediate orbital phase. Top: left panel: MECS 2-5 keV, right panel: MECS 5-10 keV; middle: left panel: HPGSPC 7-15 keV, right panel: HPGSPC 15-30 keV; bottom: left panel: PDS 13-30 keV, right panel: $30-80 \mathrm{keV}$.

was not observed in the Postapastron and Intermediate data, probably because of the lower statistics. We interpreted that feature as an absorption due to the resonant scattering of electrons in the Landau levels, i.e. a cyclotron signature. We modeled this absorption with a Gaussian filter in absorption:

$G(E)=1-\frac{E W}{\sqrt{2 \pi} \sigma} \exp \left(-\frac{\left(E-E_{\mathrm{cycl}}\right)^{2}}{2 \sigma^{2}}\right)$

where $E_{\text {cycl }}, \sigma$, and $E W$ are the centroid energy, the width, and the equivalent width of the feature, respectively. The results of the fit are reported in Table 2. In Fig. 10 (left panels) we plot the spectra and the residuals of the fit of the Preperiastron and Periastron data by using a model without the Gaussian absorption feature. The residuals clearly show that this model is unable to fit the data. In Fig. 10 (right panels) we show the spectra and the residuals of the best fit of the Preperiastron and Periastron data in which we have subtracted the Gaussian absorption feature in order to bring out its contribution to the fit. The high energy part of the spectrum turns out to be strongly affected by the cyclotron absorption.

The centroid energies are significantly different (more than $3 \sigma$ ) in the two orbital phases. This fact is more evident if we compare the spectra of the two data sets limited to the ascent of the first peak of the pulse profile (see next section), as clearly shown in Fig. 11. We find that the cyclotron energy is $\sim 53 \mathrm{keV}$ for Preperiastron data, whereas it is $\sim 45 \mathrm{keV}$ for Periastron data. Both energies are significantly higher than $E_{\text {cycl }} \sim 37 \mathrm{keV}$ reported by Makishima \& Mihara (1992) using Ginga data. But Ginga was not sensitive above $50 \mathrm{keV}$; and as it is hard to fit cyclotron features at the limit of the energy detectible range, the Ginga value could be underestimated. Using the same data of this paper, Orlandini et al. (2000) obtained very similar values. Furthermore they also reported cyclotron absorption features in the other orbital phases: Postapastron and Intermediate. 

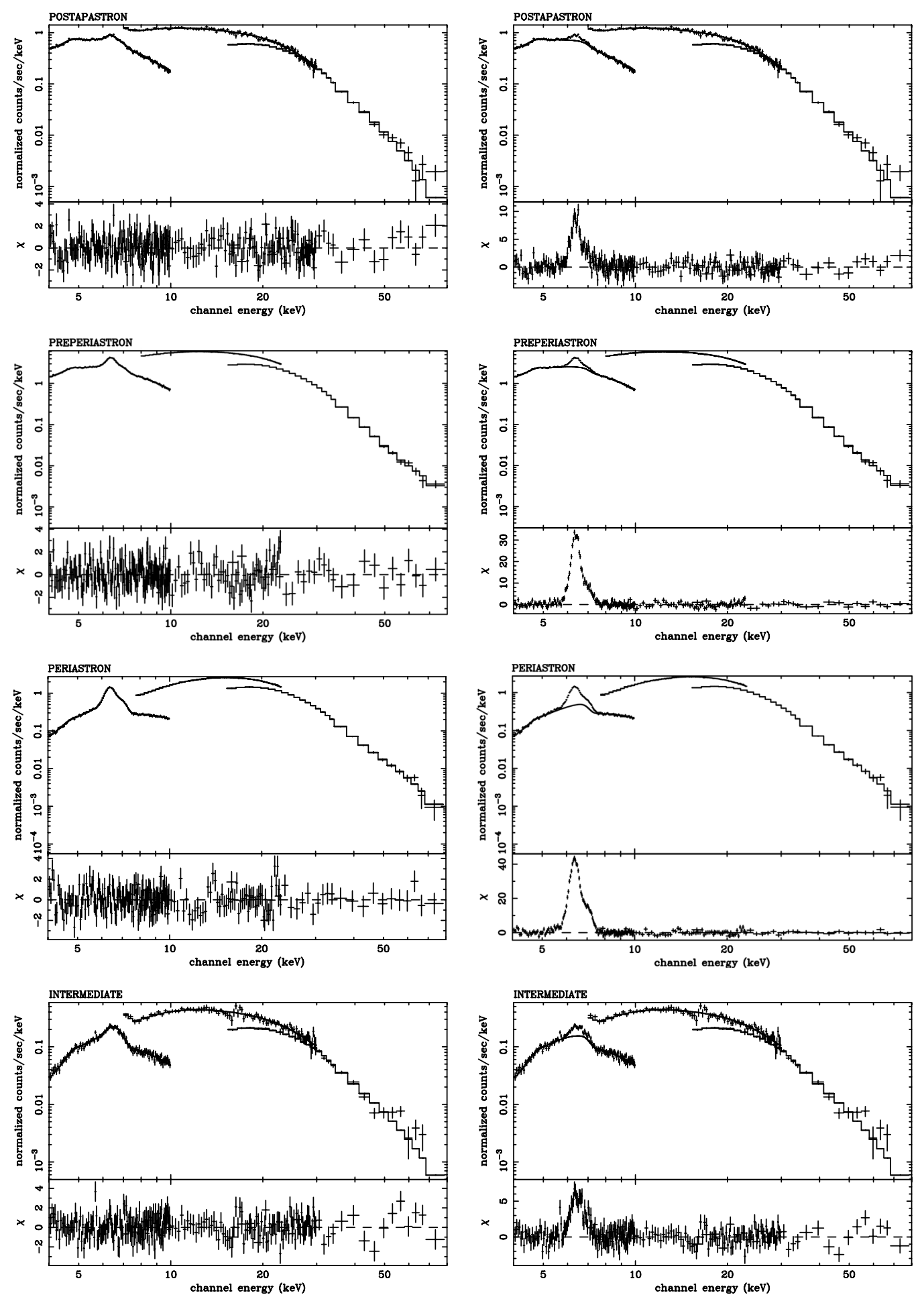

Fig. 8. BeppoSAX GX 301-2 spectra relative to the selected orbital phase intervals. In the left panels source spectra (crosses) and best fit model (histogram) are plotted together with the corresponding residuals from the best fits in $\sigma$ units. In the right panels the same spectra with the iron lines subtracted are plotted. The shape of the residuals is highly asymmetric suggesting a multicomponent structure.

The discrepancy between the results of Orlandini et al. and ours is not significant. In fact their results were preliminary; for example, they did not use the HPGSPC data, which are very important for obtaining a good constraint of the spectral continuum. Regarding the equivalent width and the $\sigma$ of the cyclotron line, these parameters vary within their uncertainties.

\section{Pulse phase resolved spectroscopy}

The statistics of Preperiastron data allowed us to perform pulse phase resolved spectroscopy of GX 301-2; however, it was not possible to study more than 4 phase intervals. We chose to analyze the ascents and the descents of both peaks of the pulse 
Table 2. Best fit results for the GX 301-2 spectra. The model used is the partial-covering absorber model corrected with a high energy cutoff plus a cyclotron absorption feature (shaped as a Gaussian in absorption) $\times$ [POW]. Two Gaussian are also added to fit the iron lines $\mathrm{K}_{\alpha}$ and $\mathrm{K}_{\beta}$. A constant factor was taken into account in order to accord MECS, HPGSPC, and PDS data. The constant was fixed to 1 for the MECS, while it was left free for HPGSPC and PDS. The uncertainties are given at $3 \sigma$ of confidence.

\begin{tabular}{|c|c|c|c|c|}
\hline Parameter & Postapastron & Preperiastron & Periastron & Intermediate \\
\hline Cycl. Eq. Width (keV) & & $22_{-12}^{+8}$ & $9_{-6}^{+9}$ & \\
\hline Cycl. En. Line (keV) & & $53_{-3}^{+1}$ & $45_{-2}^{+2.5}$ & \\
\hline Cycl. $\sigma(\mathrm{keV})$ & & $15_{-5}^{+3}$ & $10 \pm 4$ & \\
\hline$N_{\mathrm{H} 1} 10^{23} \mathrm{~cm}^{-2}$ & $2.2 \pm 0.1$ & $2.28_{-0.06}^{+0.02}$ & $4.7 \pm 0.2$ & $5.0_{-0.9}^{+0.4}$ \\
\hline$N_{\mathrm{H} 2} 10^{23} \mathrm{~cm}^{-2}$ & $19_{-8}^{+39}$ & $16_{-2}^{+1.5}$ & $11 \pm 1$ & \\
\hline Phot. index Norm & $1.35_{-0.1}^{+0.2}$ & $1.05_{-0.06}^{+0.04}$ & $0.85 \pm 0.1$ & $1.2_{-0.4}^{+0.2}$ \\
\hline Pow. Law Norm ${ }^{a}$ & $0.15_{-0.02}^{+0.04}$ & $0.31_{-0.04}^{+0.02}$ & $4.7_{-0.4}^{+1} \times 10^{-2}$ & $4_{-3}^{+2} \times 10^{-2}$ \\
\hline Pow. Law $\mathrm{Norm}_{2}{ }^{a}$ & $3.4_{-2}^{+10} \times 10^{-2}$ & $0.10 \pm 0.02$ & $0.08 \pm 0.02$ & \\
\hline$E_{\text {cut }}(\mathrm{keV})$ & $22.5 \pm 2.5$ & $20.0 \pm 0.2$ & $19.4 \pm 0.3$ & $22.5_{-3}^{+1}$ \\
\hline$E_{\text {fold }}(\mathrm{keV})$ & $9.0 \pm 0.4$ & $9.3_{-0.8}^{+0.4}$ & $8.0 \pm 0.5$ & $10.7_{-1.7}^{+0.9}$ \\
\hline Width $(\mathrm{keV})$ & $12_{-2}^{+7}$ & $11.3 \pm 0.6$ & $11.3_{-0.9}^{+0.5}$ & $15_{-4}^{+11}$ \\
\hline$E_{\mathrm{K}_{\alpha}}(\mathrm{keV})$ & $6.43 \pm 0.025$ & $6.433_{-0.004}^{+0.008}$ & $6.435 \pm 0.005$ & $6.52 \pm 0.04$ \\
\hline$\sigma_{\mathrm{K}_{\alpha}}(\mathrm{eV})$ & $<100$ & $<35$ & $<65$ & $250_{-60}^{+100}$ \\
\hline $\operatorname{Norm}_{\mathrm{K}_{\alpha}}{ }^{a}$ & $(1.36 \pm 0.15) \times 10^{-3}$ & $(1.75 \pm 0.03) \times 10^{-2}$ & $(1.49 \pm 0.5) \times 10^{-2}$ & $1.6_{-0.2}^{+0.4} \times 10^{-3}$ \\
\hline$E_{\mathrm{K}_{\beta}}(\mathrm{keV})$ & $7.0 \pm 0.1$ & $7.08 \pm 0.03$ & $7.07_{-0.06}^{+0.02}$ & \\
\hline$\sigma_{\mathrm{K}_{\beta}}(\mathrm{eV})$ & $<600$ & $<100$ & $<100$ & \\
\hline $\operatorname{Norm}_{K_{\beta}}{ }^{a}$ & $3_{-1}^{+5} \times 10^{-4}$ & $(3.35 \pm 0.3) \times 10^{-3}$ & $3.2_{-0.2}^{+0.8} \times 10^{-3}$ & \\
\hline HP Constant & $1.02 \pm 0.02$ & $1.111 \pm 0.004$ & $1.10 \pm 0.015$ & $1.21 \pm 0.05$ \\
\hline PDS Constant & $0.98 \pm 0.02$ & $0.98 \pm 0.01$ & $0.97 \pm 0.02$ & $1.04 \pm 0.04$ \\
\hline reduced $\chi^{2}$ & 1.107 & 1.185 & 1.197 & 1.099 \\
\hline d.o.f. & 232 & 199 & 201 & 237 \\
\hline
\end{tabular}

${ }^{a}$ Photons $/ \mathrm{keV} / \mathrm{cm}^{2} / \mathrm{s}$ at $1 \mathrm{keV}$.

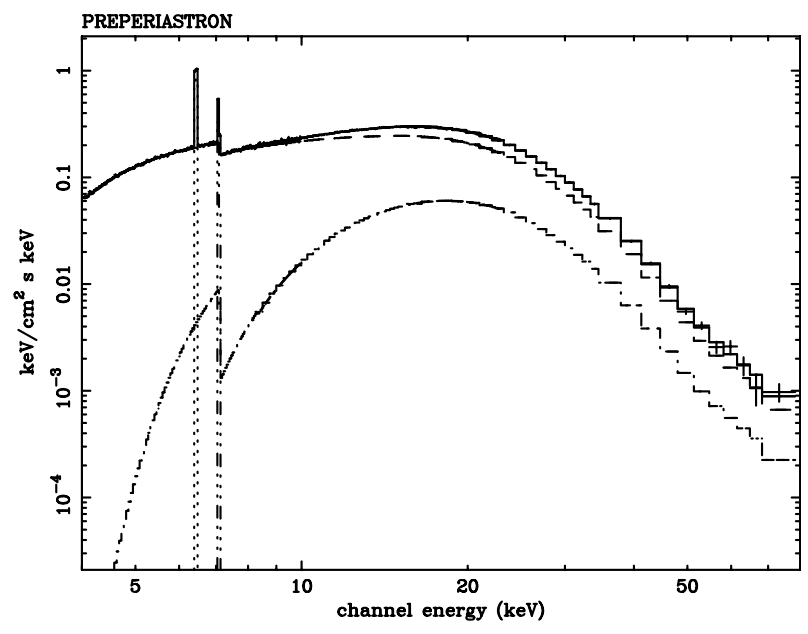

Fig. 9. Unfolded averaged spectrum of GX 301-2 relative to the Preperiastron data (crosses) superimposed on the model (solid line). The components that contribute to the model are also drawn: the less absorbed contribution to the continuum (dash-dotted line), the more absorbed component (dashed line), the $\mathrm{K}_{\alpha}$ iron fluorescence line (dotted line), the $\mathrm{K}_{\beta}$ iron fluorescence line (dash-dot-dot-dotted line).

profile as shown in Fig. 12. We refer to the peak between the pulse phases 0.0 and 0.5 as the first peak; the other between the pulse phases 0.5 and 1 will be called second peak.
We fitted the spectrum relative to each selected pulse phase interval with the same model used for the averaged spectrum and searched for variability in the spectral parameters as a function of the pulse phase. Results of the fit are reported in Table 3. In Fig. 13 the unfolded spectra relative to selected pulse phases are compared.

The low energy spectrum (below the cutoff energy) does not seem to change with the pulse phase, as the spectral index and the other low energy spectral parameters do not show significant variability. Some dependence is observed for the most absorbed hydrogen column density; it is significantly higher $\left(\mathrm{NH} \sim 40 \times 10^{23} \mathrm{~cm}^{-2}\right.$ ) during the descent of the first peak than during the ascent $\left(\mathrm{NH} \sim 11 \times 10^{23} \mathrm{~cm}^{-2}\right)$.

The high energy spectrum shows strong variability both in the energy of the cyclotron line and in the folding energy. We can roughly distinguish two phase intervals with two different behaviors. On the one hand the centroid energy of the cyclotron line has values higher than $50 \mathrm{keV}$ during the ascent of the first peak and the descent of the second peak. On the other hand, the cyclotron energy decreases below $41 \mathrm{keV}$ during the descent of the first peak and the ascent of the second, with a variation of $\sim 10 \mathrm{keV}$.

The folding energy shows similar behavior being higher during the ascent of the first peak $(9.6 \mathrm{keV})$ and the descent of the second $(7.9 \mathrm{keV})$, while it is lower during the descent of 

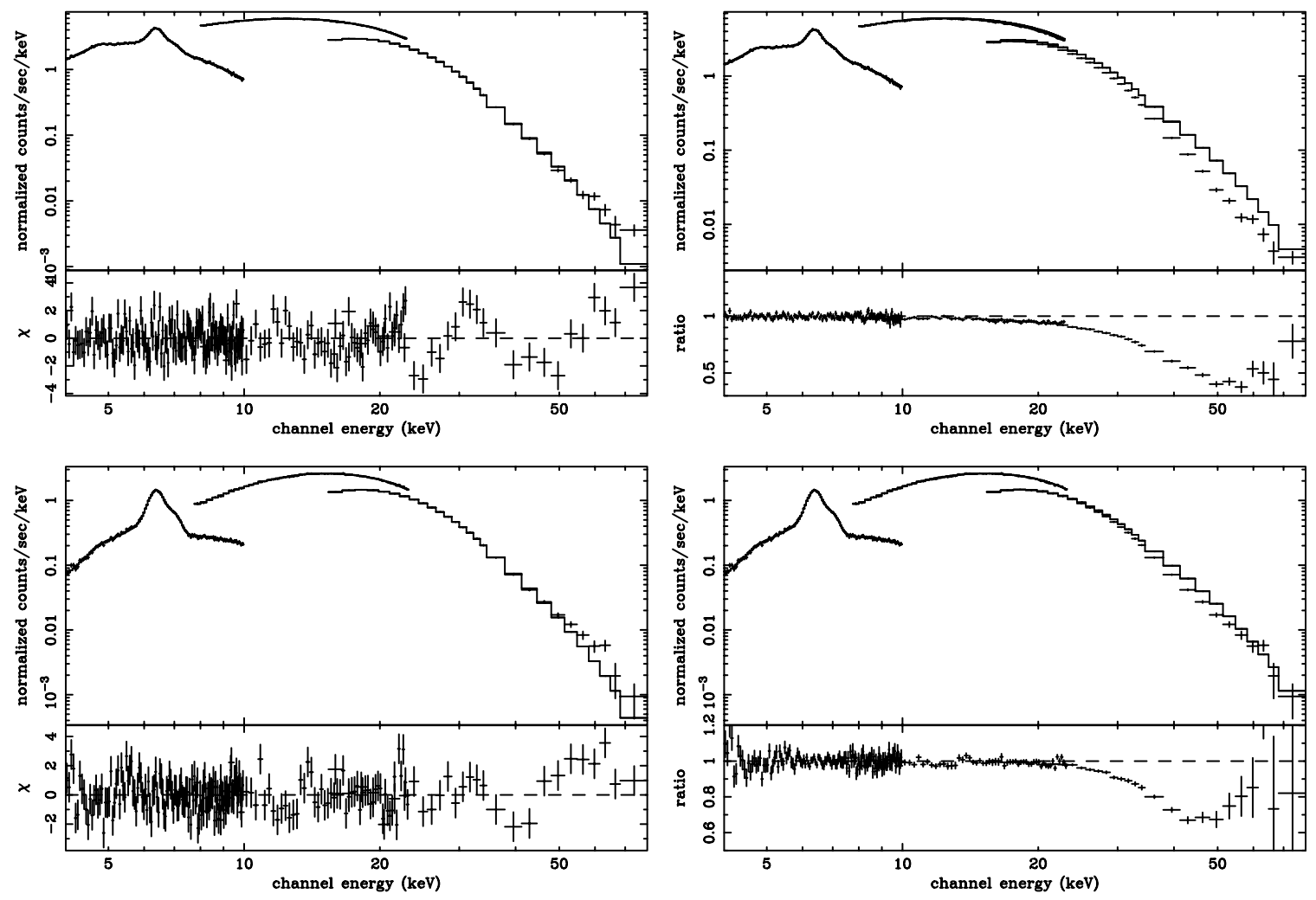

Fig. 10. Left panels: spectra and the residuals of the fit of the Preperiastron and Periastron data by using a model without the Gaussian absorption feature. Right panels: spectra and residuals of the best fit of the Preperiastron and Periastron data subtracted by the Gaussian absorption feature in order to see its contribution to the fit.

the first peak $(7.6 \mathrm{keV})$ and the ascent of the second $(7.1 \mathrm{keV})$, with a variation of $\sim 3 \mathrm{keV}$.

\section{Discussion}

The 4-80 keV spectrum of GX 301-2 was studied in great detail during 4 orbital phase intervals corresponding to 4 different levels of luminosity and accretion rate. Due to the eccentricity of the orbit, the quantity of material reaching the neutron star varies over the orbit. But this simple picture is not sufficient for describing the observations as the maximum of the luminosity is observable not during the periastron passage but at the orbital phase $\sim 0.9$. Similarly the luminosity minimum is not found at the apastron but at the orbital phase $\sim 0.2-0.3$. Several models have been introduced in order to explain the orbital light curve of GX 301-2. The formation of a gas stream orbiting and following the neutron star was introduced by Leahy (2002), and although details are not completely understood, this picture is generally accepted.

The presented BeppoSAX observations represent all the luminosity states which the source undergoes over the orbit (see Fig. 2). Postapastron experiences a flaring episode after the apastron passage; Preperiastron reveals the peak of luminosity; Periastron is representative of the periastron passage; and finally Intermediate experiences the lowest luminosity level. The large variation in luminosity causes some modifications in the spectrum of the source during its orbital motion; especially that part of the spectrum at lower energy $(E<10 \mathrm{keV})$ is affected because of the variable photoelectric absorption.
THE CONTINUUM: we fitted the spectral continuum of GX 301-2 by a power law undergoing photoelectric absorption represented by two hydrogen column densities in three groups of data: Postapastron, Preperiastron, and Periastron. This model was first introduced by Saraswat et al. (1996) interpreting the less absorbed continuum component as that one scattered by matter surrounding the pulsar. Nevertheless this interpretation has been criticized by Endo et al. (2002) because they claim to have also detected significant pulsations from the less absorbed component, indicating that the entire continuum is a direct emission from the pulsar. Probably it is not meaningful to give a simplistic interpretation of this model, individuating two separated regions responsible for the two absorbed components: one (more absorbed) closer to the neutron star surface, responsible for the production of the primary radiation, and the other (less absorbed) formed by the matter surrounding the neutron star, reflecting the primary component. The real state of the accreting plasma has to be much more complex. We can imagine that the matter is organized in blobs of different dimensions and densities because of the noisy nature of the emitted wind from the companion star, blobs which absorb the light emitted by the accretion column in different ways. Two absorptions are only an approximation of this complex environment.

The continuum of the Intermediate phase is well fitted by a power law with a single photoelectric absorption factor probably due to the low statistics, in fact this observation corresponds to the lowest level of luminosity. 


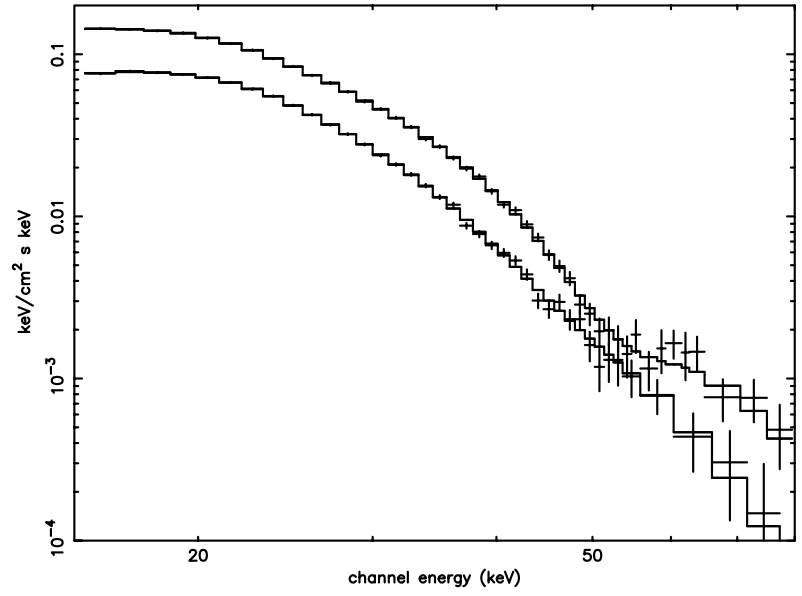

Fig. 11. Unfolded spectra of the Preperiastron (top line) and Periastron (bottom line) data obtained with PDS relative to the spin phase $0.00-0.25$ of the pulse profile (see Fig. 12). The high energy absorption feature is very different in two cases. It is stronger for Preperiastron data and centered at $\sim 53 \mathrm{keV}$, whereas it is weaker with lower energy $(\sim 47 \mathrm{keV})$ for Periastron data.

The spectral index of the power law seems to show some dependence on the orbital phase. In fact we obtain a value lower than 1 near the periastron, whereas a value larger than 1.2 far from it. Probably this difference is only an artefact of the fit, due to the fact that the low energy spectrum is strongly affected by larger photoelectric absorption near the periastron.

The continuum of all orbital phases has been corrected with a high energy exponential cutoff characterized by two parameters: $E_{\text {cut }}$ and $E_{\text {fold }}$. No evident correlation has been found between these two parameters and the orbital phase. In fact, $E_{\text {cut }}$ is consistent with a constant value of $\sim 20 \mathrm{keV}$ in all the observation groups. $E_{\text {fold }}$ shows some variation over the orbit not statistically meaningful around $\sim 9 \mathrm{keV}$. Generally the high energy cutoff correction is believed to be a result of the Comptonization of the original radiation. In this frame $E_{\text {fold }}$ is interpreted as the temperature of the electrons in the accretion column. The ratio $E_{\text {cut }} / E_{\text {fold }}<3$ suggests an unsaturated Comptonization.

Makishima \& Mihara (1992) have suggested a correlation between $E_{\text {cut }}$ and the energy of the cyclotron line $E_{\text {cycl }}$ in the form $E_{\text {cycl }} \simeq 2 E_{\text {cut }}$, indicating that the cutoff energy is related to the cyclotron resonant scattering. Calculating this ratio, we obtained $2.6 \pm 0.1$ in the case of Preperiastron and $2.3 \pm 0.1$ in the case of Periastron. This result, together with the fact that we found that the cyclotron energy is correlated with the luminosity of the source, whereas $E_{\text {cut }}$ does not show evidence of this correlation, makes the correlation claimed by Makishima and Mihara in its present simple form doubtful.

THE IRON LINES: we have clearly resolved both $\mathrm{K}_{\alpha}$ at $\sim 6.4 \mathrm{keV}$ and $\mathrm{K}_{\beta}$ at $\sim 7 \mathrm{keV}$ emission lines due to the iron fluorescence in Preperiastron and Periastron data. There is also marginal evidence of $\mathrm{K}_{\beta}$ in Postapastron data. No evidence of the $\mathrm{K}_{\beta}$ line is found in the Intermediate phase probably because of the low statistics of the data. Unfortunately the MECS is

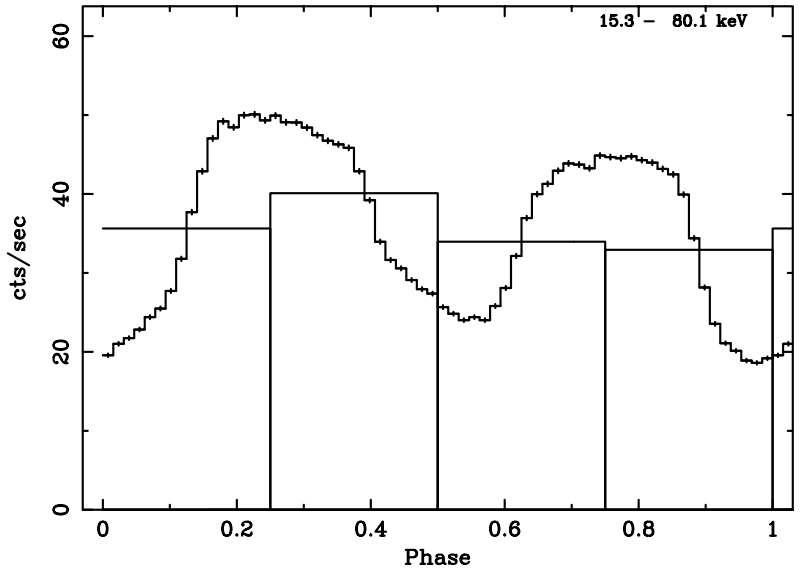

Fig. 12. Selected pulse phase intervals superimposed on the PDS pulse profile: ascent of the first peak $(0.00-0.25)$, descent of the first peak $(0.25-0.50)$, ascent of the second peak $(0.50-0.75)$, descent of the second peak $(0.75-1.00)$.

unable to resolve the width of the lines and only upper limits are possible. Endo et al. (2002), analyzing ASCA data near the periastron, report a value for sigma of the $\mathrm{K}_{\alpha}$ of $79 \pm 6 \mathrm{eV}$. We obtain $\sigma_{\alpha} \leq 35 \mathrm{eV}$ in Preperiastron data and $\leq 65 \mathrm{eV}$ in Periastron data. The value of Preperiastron is significantly smaller than that reported by Endo et al., but probably a comparison is not possible because $\sigma$ is strongly dependent on the orbital phase. In fact the value obtained in the Periastron data is very close to that reported by those authors.

Several studies performed on a large number of X-ray sources have shown a strong correlation between the equivalent width $(E W)$ of the iron line and the hydrogen column density when $N_{\mathrm{H}}>10^{23} \mathrm{~cm}^{-2}$. Inoue (1985) derived the expected equivalent width of the iron $\mathrm{K}_{\alpha}$ line as a function of the hydrogen column density. For cosmic abundances, in a spherical geometry, and for a photon index of 1.1, he derived:

$E W=100\left(N_{\mathrm{H}} / 10^{23}\right) \mathrm{eV}$.

For Preperiastron and Periastron we obtained $E W \mathrm{~s}$ of $400 \mathrm{eV}$ and $1500 \mathrm{eV}$ respectively. Adopting these results we expect values of the Hydrogen column density ranging between about $4 \times 10^{23} \mathrm{~cm}^{-2}$ (Preperiastron) and $15 \times 10^{23} \mathrm{~cm}^{-2}$ (Periastron), within a factor 2-3 with respect to the values obtained by the fit.

In the case of Intermediate data a single Gaussian centered at $\sim 6.5 \mathrm{keV}$ was sufficient to fit the data. For this line we obtained a $\sigma$ of $250 \mathrm{eV}$ much larger than those measured in the other orbital phases. This discrepancy is due to the single Gaussian modelling both the $\mathrm{K}_{\alpha}$ and the $\mathrm{K}_{\beta}$ iron lines. The $\mathrm{K}_{\beta}$ line could not be resolved because of the low statistics of the data.

THE CYCLOTRON LINE: we found an absorption feature at higher energies in the data taken close to the periastron passage. To fit the feature, we used a Gaussian in absorption determining the energy of the centroid, the width of the Gaussian, and the equivalent width. In particular, the Preperiastron data show the feature centered at $\sim 53 \mathrm{keV}$, whereas the Periastron data show a similar feature centered at $\sim 45 \mathrm{keV}$. The difference between 
Table 3. Fit results of spectra of GX 3001-2 relative to the selected pulse phases obtained from the Preperiastron data. The model used is the partial-covering absorber model corrected with a high energy cutoff plus a cyclotron absorption feature. The uncertainties are given at $3 \sigma$ of confidence.

\begin{tabular}{|c|c|c|c|c|}
\hline Parameter & First ascent & First descent & Second ascent & Second descent \\
\hline Cycl. Eq. Width (keV) & $11_{-3}^{+8}$ & $2.5_{-1}^{+2}$ & $5_{-4}^{+9}$ & $77_{-3}^{+11}$ \\
\hline Cycl. En. Line (keV) & $52_{-1.5}^{+2.5}$ & $41_{-2}^{+1}$ & $40_{-2}^{+4}$ & $51_{-3}^{+4}$ \\
\hline Cycl. $\sigma(\mathrm{keV})$ & $7 \pm 2$ & $4 \pm 2$ & $9 \pm 4$ & $6.5 \pm 3$ \\
\hline$N_{\mathrm{H} 1} 10^{23} \mathrm{~cm}^{-2}$ & $2.3 \pm 0.1$ & $2.2_{-0.05}^{+0.1}$ & $2.2 \pm 0.7$ & $2.4 \pm 0.1$ \\
\hline$N_{\mathrm{H} 2} 10^{23} \mathrm{~cm}^{-2}$ & $11_{-2.5}^{+2}$ & $40 \pm 4$ & $27_{-9}^{+5}$ & $12 \pm 3$ \\
\hline Phot. index Norm & $1.2 \pm 0.1$ & $1.06 \pm 0.05$ & $0.86_{-0.06}^{+0.1}$ & $0.97_{-0.2}^{+0.07}$ \\
\hline Pow. Law Norm ${ }_{1}^{a}$ & $0.44_{-0.03}^{+0.08}$ & $0.30_{-0.03}^{+0.04}$ & $0.18_{-0.015}^{+0.03}$ & $0.21 \pm 0.04$ \\
\hline Pow. Law Norm ${ }_{2}^{a}$ & $0.2_{-0.05}^{0.1}$ & $0.2 \pm 0.1$ & $0.09_{-0.03}^{+0.07}$ & $0.07_{-0.01}^{+0.03}$ \\
\hline$E_{\text {cut }}(\mathrm{keV})$ & $19.8 \pm 0.4$ & $19.5 \pm 1$ & $19 \pm 1$ & $19.7_{-0.1}^{+0.3}$ \\
\hline$E_{\text {fold }}(\mathrm{keV})$ & $9.6_{-0.2}^{+1}$ & $7.6_{-0.3}^{+0.2}$ & $7.1_{-0.4}^{+0.5}$ & $7.9_{-0.3}^{+0.5}$ \\
\hline Width (keV) & $9.2 \pm 0.5$ & $17_{-2}^{+4}$ & $17.0_{-3}^{+6}$ & $10 \pm 1$ \\
\hline$E_{\mathrm{K}_{\alpha}}(\mathrm{keV})$ & $6.43_{-0.02}^{+0.01}$ & $6.43 \pm 0.01$ & $6.43 \pm 0.01$ & $6.45_{-0.02}^{+0.01}$ \\
\hline$\sigma_{\mathrm{K}_{\alpha}}(\mathrm{eV})$ & $<85$ & $<65$ & $<70$ & $<100$ \\
\hline $\operatorname{Norm}_{\mathrm{K}_{\alpha}}{ }^{a}$ & $1.7 \pm 0.1 \times 10^{-2}$ & $(1.7 \pm 0.1) \times 10^{-2}$ & $(1.8 \pm 0.1) \times 10^{-2}$ & $1.7 \pm 0.1 \times 10^{-2}$ \\
\hline$E_{\mathrm{K}_{\beta}}(\mathrm{keV})$ & $7.1 \pm 0.1$ & $7.05 \pm 0.05$ & $7.07 \pm 0.05$ & $7.1_{ \pm} 0.1$ \\
\hline$\sigma_{\mathrm{K}_{\beta}}(\mathrm{eV})$ & $<200$ & $<100$ & $<200$ & $<400$ \\
\hline Norm $_{K_{\beta}}{ }^{a}$ & $(4 \pm 1) \times 10^{-3}$ & $(2 \pm 1) \times 10^{-3}$ & $(4 \pm 1) \times 10^{-3}$ & $(4 \pm 1) \times 10^{-3}$ \\
\hline HP Constant & $1.17 \pm 0.02$ & $1.10 \pm 0.01$ & $1.09 \pm 0.02$ & $1.13 \pm 0.02$ \\
\hline PDS Constant & $1.00 \pm 0.02$ & $0.95 \pm 0.01$ & $0.99_{-0.01}^{+0.02}$ & $1.01 \pm 0.02$ \\
\hline reduced $\chi^{2}$ & 1.023 & 1.116 & 1.000 & 1.114 \\
\hline d.o.f. & 227 & 227 & 226 & 227 \\
\hline
\end{tabular}

${ }^{a}$ Photons $/ \mathrm{keV} / \mathrm{cm}^{2} / \mathrm{s}$ at $1 \mathrm{keV}$.

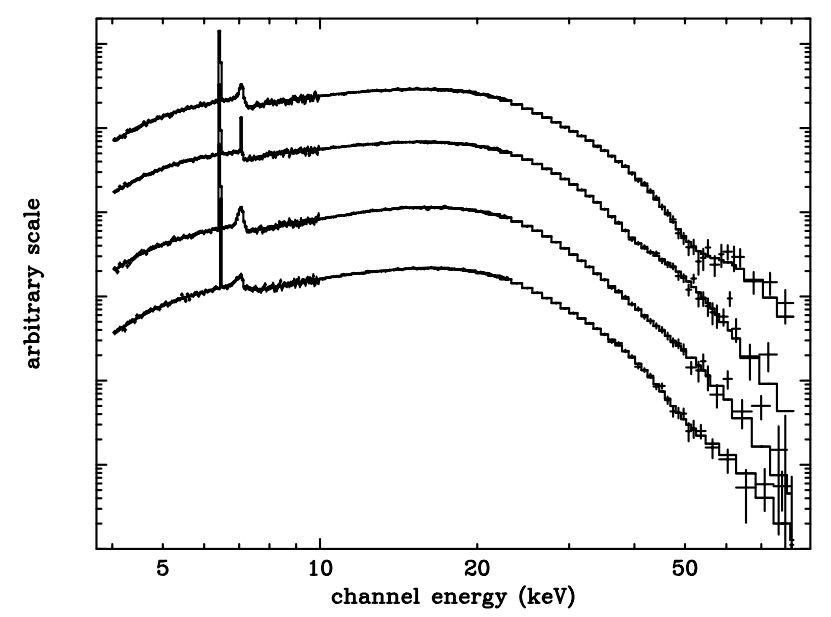

Fig. 13. Unfolded spectra $\left(\mathrm{keV} /\left(\mathrm{cm}^{2} \mathrm{~s} \mathrm{kev}\right)\right)$ of GX $301-2$ relative to the selected pulse phases obtained from the Preperiastron data. From the top: ascent of the first peak, descent of the first peak, ascent of the second peak, descent of the second peak. The data have been scaled for clarity.

these two values is significant and seems to be independent of the continuum model that we tried. We interpreted these features as CRSFs. In this model, taking the gravitational redshift effect into account, the magnetic field strength is obtained by the cyclotron energy through the simply relation:

$B_{12}=\frac{1}{\sqrt{1-\frac{2 G M_{\mathrm{NS}}}{R c^{2}}}} \frac{E_{\mathrm{cycl}}}{11.6}$ where $B_{12}$ is the magnetic field in $10^{12}$ Gauss units, $E_{\text {cycl }}$ the observed cyclotron centroid energy in $\mathrm{keV}, G$ the gravitational constant, $M_{\mathrm{NS}}$ the neutron star mass, $R$ its radius, and $c$ the velocity of the light. Adopting the neutron star mass of $1.4 M_{\odot}$ and a Neutron star radius of $10^{6} \mathrm{~cm}$, we derive magnetic field values ranging from $\sim 5.1 \times 10^{12}$ to $\sim 5.8 \times 10^{12}$ Gauss.

Variation in the centroid energy of the CRSF observed at two different orbital phases could depend on the variation of the luminosity with the orbit. If this is the case, we would have a direct correlation between the cyclotron energy and the luminosity level of the source. In fact we observe a higher cyclotron energy at the orbital phase where the luminosity is also higher.

Some experimental evidence (see e.g. Burderi et al. 2000; La Barbera et al. 2003) suggests that the cyclotron emission region is very close to the polar caps of the neutron star within a characteristic length of few hundreds meters (see e.g. Wu et al. 1999). The cyclotron energy $E_{\text {cycl }}$ is strongly dependent on the mean magnetic field $B$ which characterize the emission region: $E_{\text {cycl }} \propto B$. Assuming a NS magnetic field with dipolar structure, we expect that the regions with higher values of magnetic field are those closer to the surface of the star. This means that during the orbital phase Preperiastron we access an emission region closer to the neutron star surface than during the orbital phase Periastron. The interpretation of this result strongly depends on the structure of the accretion column.

Unfortunately there is not a unique, self consistent model of the accretion column. It is known that for luminosities $L_{\mathrm{x}}>$ $10^{37} \mathrm{erg} \mathrm{s}^{-1}$, the radiation pressure, by decelerating the accreting matter, plays a dominant role in determining the structure 
of the accreting column. On the other hand, for luminosities $L_{\mathrm{x}}<10^{37}$ the physics is less clear. In this case several physical mechanisms are concurrent in deceleration of the infalling plasma: Coulomb collisions, inelastic nuclear collisions, or formation of collisionless shock (see e.g. Harding et al. 1984).

According to the radiation dominated models (see e.g. Basko \& Syunaiev 1976) the height $H$ of the accretion column is correlated with the accretion rate $\dot{M}$. In first approximation we can write:

$H \propto \dot{M}$.

Under the assumption that the emission region coincides with the shock in the accretion column, we should observe cyclotron lines at lower energy when the source is in a higher luminosity state. This result contrasts with what we observe in GX 301-2.

On the other hand, for low accretion regimes $\left(L_{\mathrm{X}}<\right.$ $10^{37} \mathrm{erg} / \mathrm{s}$ ), collisions among the particles dominate the accretion physics and, also in this case, an accretion column can be formed (see e.g. Harding et al. 1984). Making some simple assumptions it is possible to obtain the relation:

$\dot{M} \propto \frac{M_{\text {col }}}{H}$,

where $M_{\mathrm{col}}$ is the mass of the gas contained into the accretion column. According to Eq. (6), if $M_{\text {col }}$ depends weakly on the accretion rate and on the height of the accretion column, $\dot{M}$ and $H$ have an inverted dependence with respect to the case of the radiation dominated accretion column. The major problem of these models is that the scale height of the accretion column is only 10-200 cm (see e.g. Harding et al. 1984) which is not able to explain the observed variations of the magnetic field intensity.

In conclusion, the relation between the cyclotron energy and the luminosity suggested by our data cannot be explained by one-dimensional models of the accretion column, because the radiation dominated accretion column implies the inverted correlation between height of the accretion column and accretion rate. On the other hand the gas pressure dominated accretion column, even if it gives the correct dependence, is not able to form a sufficient high accretion column to explain other important phenomenological results. In addition, it is impossible to decide what kind of accretion regime is involved on the surface of GX 301-2. In fact by assuming a distance of $1.8 \mathrm{kpc}$ (Parkes et al. 1980) the resulting luminosity $(2-100 \mathrm{keV})$ is $2 \times 10^{37} \mathrm{erg} / \mathrm{s}$ for Preperiastron data and $8 \times 10^{36} \mathrm{erg} / \mathrm{s}$ for Periastron data, which would suggest a low accretion regime. On the other hand, assuming a distance of $5.3 \mathrm{kpc}$ (Kaper et al. 1995), the resulting luminosity is about an order of magnitude higher in both cases which suggests a high accretion regime. Probably, to explain our problematic relation between cyclotron energy and luminosity state, other effects should be considered as, for instance, the gradient of the magnetic field along the direction of the neutron star surface and not only in the vertical direction. Unfortunately the structure of the accretion column along the horizontal direction is less understood.

The width of the cyclotron line was observed to range between $\sim 10 \mathrm{keV}$ and $\sim 15 \mathrm{keV}$. These values could be explained by thermal broadening. Assuming a mean temperature $E_{\text {fold }}$ of $8 \mathrm{keV}$ (obtained by the fit) and a cyclotron energy $E_{\text {cycl }}$ of $50 \mathrm{keV}$, we expect a mean broadening of:

$\Delta E_{\text {cycl }}=E_{\text {cycl }} \sqrt{E_{\text {fold }} / 511 \mathrm{keV}} \simeq 6 \mathrm{keV}$

within a factor 2 with respect to the values obtained by the fit. The same broadening could be attributed to the distribution of the values of the magnetic field strength in the emission region. In this case the expected broadening is at least on the order of the $\sim \Delta E_{\text {cycl }}$. Considering the range of values spanned by the measured cyclotron energy, we obtain $\Delta E_{\text {cycl }} \sim 8 \mathrm{keV}$. Doppler broadening, due to the infalling velocity of the accretion material, could also contribute the broadening of the cyclotron line. In fact near the neutron star the infalling velocity can be a significant fraction of the light speed $c$ with values on the order of $0.1 c$ (see e.g. Wu et al. 1999). Adopting this result we expect a broadening on the order of $\Delta E_{\text {cycl }} \sim 0.1 E_{\text {cycl }} \sim 5 \mathrm{keV}$, about the same as the other effects.

PHASE RESOLVED SPECTROSCOPY: phase resolved spectroscopy performed on 4 pulse phase intervals of the Preperiastron data shows a significant variation both in the energy of the cyclotron line centroid and in the folding energy (see Fig. 14). Furthermore a correlation between the behavior of the cyclotron energy and that of the folding energy is suggested. In fact in the phase intervals $0.00-0.25$ and $0.75-1.00$, the cyclotron energy is higher than $50 \mathrm{keV}$ and the folding energy ranges between 7.9 and $9.6 \mathrm{keV}$. On the other hand, in the phase intervals $0.25-0.50$ and $0.50-0.75$, the cyclotron energy has values lower than $41 \mathrm{keV}$ and the folding energy ranges between 7.1 and $7.6 \mathrm{keV}$.

As previously explained, the cyclotron energy is a measure of the magnetic field present in the emission region. Assuming a dipolar magnetic field, higher magnetic field values correspond to emission regions closer to the surface of the neutron star and then to regions with higher plasma temperature. In this frame the variation in the cyclotron energy with the pulsation has to be attributed to the fact that different emission regions with different magnetic fields are visible. Assuming that the accretion column has a vertical structure, as suggested by several authors (see e.g. Wu et al. 1999, and references therein), we could attribute the phase dependent luminosity to the darkening limb effect due to the different geometrical depths seen. According to this, different phases allow access to different heights of the accreting column and then to different values of magnetic field strength. In this context, interpreting the folding energy as the temperature of the electrons in the accretion column, a correlated behavior of the cyclotron energy with the folding energy is expected.

The cyclotron energy shows an asymmetric behavior with respect to the pulse profile. This can be interpreted by simply assuming that the magnetic dipole of the neutron star is displaced with respect to its geometric center. This asymmetry, observed in other sources (see e.g. Burderi et al. 2000; La Barbera et al. 2003) seems to be a general characteristic of the high mass X-ray pulsars. 

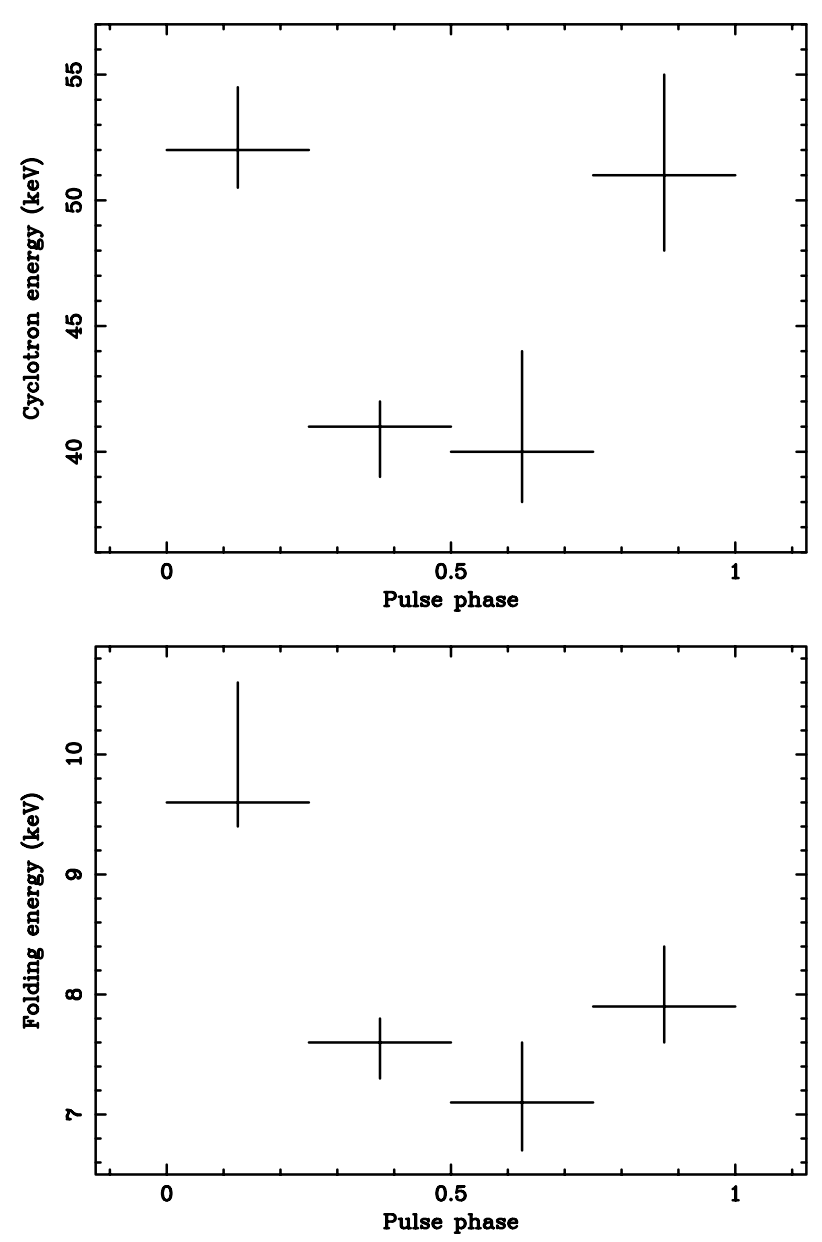

Fig. 14. The centroid energy of the cyclotron absorption feature (top panel) and the folding energy (bottom panel) as a function of the pulse phase.

\section{Conclusions}

We have studied the 4-80 keV spectrum of GX 301-2 observed by BeppoSAX in 4 orbital phase intervals, and confirm that a single power law with a single photoelectric absorption factor is insufficient describing the low energy continuum. Therefore, to fit the low energy spectral continuum, we adopted the partialcovering absorber model. Some caution has to be taken in interpreting the results of this model. In fact, distinguishing two precise regions responsible for the two photoelectric absorptions is probably meaningless. Comptonization processes could well explain the high energy cutoff that does not show any evident correlation with the luminosity level of the source.

Both $\mathrm{K}_{\alpha}$ at $\sim 6.4 \mathrm{keV}$ and $\mathrm{K}_{\beta}$ at $\sim 7 \mathrm{keV}$ emission lines due to the iron fluorescence have been resolved. The resulting line parameters are consistent with those obtained by Endo et al. (2002) based on ASCA data, who also report an iron edge at $\sim 7 \mathrm{keV}$. No evidence of iron edge is present in our data.

A cyclotron resonance absorption feature showing dependence on the orbital motion was observed in data taken close to the periastron passage. In particular a cyclotron energy of $45 \mathrm{keV}$ was observed at the periastron (phase 0.0), while a value of $53 \mathrm{keV}$ was obtained at orbital phase 0.9 . A comparison of this result with the trend of the luminosity over the orbit could suggests direct correlation between the cyclotron energy and luminosity state of the source, even if other observations would be needed to verify this as fact. Nevertheless, if confirmed, interpretation of this dependence would be problematic. Present one-dimensional models of accretion column have some difficulty fitting the observational results as both radiation pressure dominated models and matter dominated models fail to explain the phenomenological results for different reasons. Probably a multidimensional model of accretion column is needed, but unfortunately so far there is no consistent accretion column model able to describe its three-dimensional structure.

Phase-resolved spectroscopy performed on data taken at the orbital phase about 0.9 shows a dependence of the cyclotron energy and of the folding energy on the X-ray pulsation. Their observed behavior suggests that these two quantities are correlated. The observational results are consistent with an accretion column with a vertical structure dominated by a dipolar magnetic field. The fact that the variation in the cyclotron line is asymmetric with respect to the pulse profile suggests that the magnetic dipole is displaced with respect to the geometric center of the NS as observed in other HMXBs.

Acknowledgements. This research was funded in part by the Italian Space Agency, and was also developed thanks to the Short Term Mobility program of the Italian "Consiglio Nazionale delle Ricerche", which sustained the collaboration with the IAA of the University of Tuebingen.

\section{References}

Araya, R. A., \& Harding, A. K. 1999, ApJ, 517, 334

Araya-Góchez, R. A., \& Harding, A. K. 2000, ApJ, 544, 1067

Basko, M. M., \& Sunyaev, R. A. 1976, MNRAS, 175, 395

Blondin, J. M., Kallman, T. R., Fryxell, B. A., \& Taam, R. E. 1990, ApJ, 356, 591

Blondin, J. M., Stevens, I. R., \& Kallman, T. R. 1991, ApJ, 371, 684

Boella, G., Butler, R. C., Perola, G. C., et al. 1997a, A\&AS, 122, 299

Boella, G., Chiappetti, L., Conti, G., et al. 1997b, A\&AS, 122, 327

Burderi, L., Di Salvo, T., Robba, N. R., La Barbera, A., \& Guainazzi, M. 2000, ApJ, 530, 429

Endo, T., Ishida, M., Masai, K., et al. 2002, ApJ, 574, 879

Fiore, F., Guainazzi, M., \& Grandi, P. 1999, Cookbook for BeppoSAX NFI spectral analysis, in http://www .asdc.asi.it/bepposax/software/cookbook

Frontera, F., Costa, E., dal Fiume, D., et al. 1997, A\&AS, 122, 357

Frontera, F., \& dal Fiume, D. 1989, in 23rd Symposium on Two Topics in X-ray Astronomy, 57, ESA SP-297

Haberl, F. 1991, ApJ, 376, 245

Harding, A. K., Mészáros, P., Kirk, J. G., \& Galloway, D. J. 1984, ApJ, 278, 369

Inoue, H. 1985, Space Sci. Rev., 40, 317

Jager, R., Mels, W. A., Brinkman, A. C., et al. 1997, A\&AS, 125, 557

Kaper, L., Lamers, H. J. G. L. M., Ruymaekers, E., et al. 1995, A\&A, 300, 446

Koh, D. T., Bildsten, L., Chakrabarty, D., et al. 1997, ApJ, 479, 933

Kreykenbohm, I., et al. 2004, A\&A, submitted

La Barbera, A., Santangelo, A., Orlandini, M., \& Segreto, A. 2003, A\&A, 400, 993

Leahy, D. A., Nakajo, M., Matsuoka, M., et al. 1988, PASJ, 40, 197

Leahy, D. A. 2002, A\&A, 391, 219 
Lyubarskii, Y. E., \& Syunyaev, R. A. 1988, Sov. Astron. Lett., 14, 390

Makishima, K., \& Mihara, T. 1992, in Magnetic Fields of Neutron Stars, ed. Y. Tanaka, \& K Koyama (Tokyo: Universal Academy Press), 23

Manzo, G., Giarrusso, S., Santangelo, A., et al. 1997, A\&AS, 122, 341

Meszaros, P., \& Nagel, W. 1985a, ApJ, 298, 147

Meszaros, P., \& Nagel, W. 1985b, ApJ, 299, 138

Mihara, T. 1995, Ph.D. Thesis, University of Tokyo

Mihara, T., Makishima, K., \& Nagase, F. 1998, Adv. Space Res., 22, 987

Nagel, W. 1981, ApJ, 251, 278

Orlandini, M., dal Fiume, D., Frontera, F., et al. 2000, Adv. Space Res., 25, 417

Parkes, G. E., Culhane, J. L., Mason, K. O., \& Murdin, P. G. 1980, MNRAS, 191, 547
Parmar, A. N., Martin, D. D. E., Bavdaz, M., et al. 1997, A\&AS, 122, 309

Pravdo, S. H., Day, C. S. R., Angelini, L., et al. 1995, ApJ, 454, 872

Pravdo, S. H., \& Ghosh, P. 2001, ApJ, 554, 383

Saraswat, P., Yoshida, A., Mihara, T., et al. 1996, ApJ, 463, 726

Sato, N., Nagase, F., Kawai, N., et al. 1986, ApJ, 304, 241

White, N. E., Mason, K. O., Huckle, H. E., Charles, P. A., \& Sanford, P. W. 1976, ApJ, 209, L119

White, N. E., Mason, K. O., \& Sanford, P. W. 1978, MNRAS, 184, 67

White, N. E., Swank, J. H., \& Holt, S. S. 1983, ApJ, 270, 711

White, N. E., \& Swank, J. H. 1984, ApJ, 287, 856

Watson, M. G., Warwick, R. S., \& Corbet, R. H. D. 1982, MNRAS, 199, 915

Wu, H., Lin, X., Xu, H., \& You, J. 1999, ChA\&A,23, 498 